\title{
PRÁCTICAS DE ACOMPAÑAMIENTO DE PROFESORES TUTORES EN CARRERAS DE PEDAGOGÍA: ANÁLISIS DE LOS DISPOSITIVOS DE ASEGURAMIENTO Y CERTIFICACIÓN DE LA CALIDAD DE LOS APRENDIZAJES TERMINALES ${ }^{1}$
}

\author{
Marisol Latorre ${ }^{2}$, Claudia Vergara ${ }^{3}$, María Alejandra \\ Morales $^{4}$, Alejandra Orbeta ${ }^{5}$, Claudia Escobar ${ }^{6}$, \\ Marta Quiroga ${ }^{7}$
}

\begin{abstract}
RESUMEN
En los últimos 25 años, la tradicional formación teórica, académica y disciplinar ha ido dando paso a perspectivas y orientaciones teórico-prácticas y pedagógico-didácticas que se han incorporado con fuerza en el currículo de formación inicial docente, posicionando la formación práctica como uno de los componentes claves. Además, en el marco del Sistema de Aseguramiento de la Calidad y los procesos de acreditación, los perfiles de egreso y los aprendizajes propuestos en ellos se han vuelto el foco de atención, tensionando la coherencia de los planes de estudio y las experiencias formativas respecto de los logros esperados. El presente estudio investiga cómo dialoga la formación de las prácticas finales con el aseguramiento y certificación de los aprendizajes comprometidos en los perfiles de egreso de las carreras de pedagogía. Para lograr este objetivo se utilizó un enfoque de investigación exploratorio-descriptivo y comprensivo, con un diseño de estudio de casos múltiples que profundiza en doce programas de dos universidades de la zona central de Chile. Los resultados reportan: limitada presencia de las actividades prácticas en los currículos de formación docente, que no supera el 10\%; rol periférico, ambiguo y sobrecargado de los tutores de práctica final; las reuniones del estudiante en práctica con el tutor abordan mayoritariamente temas idiosincráticos; el acompañamiento que el tutor entrega al estudiante en práctica se traduce en una presencia constante en la escuela.
\end{abstract}

Conceptos clave: formación práctica en carreras de pedagogía, formación inicial docente, tutorías en formación de profesores, aprendizajes prácticos en formación inicial docente.

Proyecto apoyado financieramente por CNED/ Convocatoria 2017.

Universidad Alberto Hurtado, Santiago, Chile. Contacto: mlatorrn@uahurtado.cl

Universidad Alberto Hurtado, Santiago, Chile. Contacto: clvergara@uahurtado.cl

Universidad Alberto Hurtado, Santiago, Chile. Contacto: mamoralg@uahurtado.cl

Universidad Alberto Hurtado, Santiago, Chile. Contacto: aorbeta@uahurtado.cl

Universidad Alberto Hurtado, Santiago, Chile. Contacto: cpescob@uahurtado.cl

Pontificia Universidad Católica de Valparaíso, Valparaíso, Chile.

Contacto: marta.quiroga@pucv.cl 


\title{
ACCOMPANYING PRACTICES FOR TUTORS IN TEACHING PROGRAMS: ANALYSIS OF THE QUALITY ASSURANCE AND CERTIFICATION DEVICES FOR TERMINAL LEARNING
}

\begin{abstract}
In the last 25 years, the traditional theoretical, academic, and disciplinary education has led to theoretical-practical and pedagogical perspectives and orientations that have been strongly incorporated into the curriculum of initial teacher's training, positioning the practical training as one of the key components. On the other hand, within the framework of the System of Quality Assurance and the accreditation processes, the graduation profiles and the learning objectives proposed in it, have become the center of attention, stressing the coherence of the study plans and formative experiences regarding the expected achievements. This study investigates how training at the final internship dialogues with the assurance and certification of the learning related to the graduate profiles of teacher education programs. To achieve this objective, an exploratory-descriptive and comprehensive research approach was used, with a multiple case studies design that delves into twelve teaching programs in two universities in central Chile. The results report: limited presence of practical activities in teacher training curriculum which is less than 10\%; a peripheral, ambiguous and overloaded role of final internship tutors; meetings between interns and tutors mainly address idiosyncratic topics; the guideline that the tutor gives the intern means a constant presence at school.
\end{abstract}

Key concepts: practical training in educational programs -initial teacher's training-tutorial in final teacher's training - inicial internship training in teaching educational programs 
184 PRÁCTICAS DE ACOMPAÑAMIENTO DE PROFESORES TUTORES EN CARRERAS DE PEDAGOGÍA: ANÁLISIS DE LOS DISPOSITIVOS DE ASEGURAMIENTO Y CERTIFICACIÓN DE LA CALIDAD DE LOS APRENDIZAJES TERMINALES - M. Latorre, C. Vergara, M. Morales, A. Orbeta, C. Escobar, M. Quiroga

\section{Introducción}

En el marco del Sistema de Aseguramiento de la Calidad y de los procesos de acreditación de carreras, los perfiles de egreso se han vuelto foco de atención e interés creciente. Recientemente, la nueva política de carrera docente plantea, entre otras exigencias, la incorporación de la Evaluación Nacional Diagnóstica (END), que deben rendir todos los estudiantes de pedagogía en el penúltimo año de su formación y que es requisito para su titulación y graduación. En este contexto, disponer de información específica acerca de los mecanismos de evaluación y certificación de aprendizajes terminales de carreras de pedagogía, y de las acciones de formación asociadas a ellos, resulta estratégico para abordar las debilidades que se evidencien y evaluar los resultados de los planes de fortalecimiento que se implementen, y para atenderlas y asegurar el logro efectivo de los aprendizajes comprometidos en los perfiles de egreso de cada carrera.

Comprender y analizar la realidad educativa desde la perspectiva del docente; problematizar las dinámicas de la escuela; dominar los conocimientos didácticos y pedagógicos asociados a la enseñanza y el aprendizaje de una disciplina específica; atender a los procesos cotidianos de deliberación profesional; imaginar y desarrollar alternativas de cambio y mejora, son, entre otros, algunos de los aprendizajes fundamentales involucrados en los desempeños profesionales de un futuro profesor. En la base de estos desempeños están las trayectorias formativas desarrolladas por cada estudiante, en las que ciclos, áreas y líneas de formación se organizan para representar, de manera coherente, las relaciones de un conjunto diverso de conocimientos y saberes, teóricos y prácticos, propios del campo disciplinar y del campo pedagógico.

La tradicional preeminencia de la formación teórica, académica y disciplinar ha ido dando paso a una formación más bien teóricopráctica con orientación pedagógica-didáctica, y, en los últimos años, el componente práctico se ha incorporado con fuerza en el currículo de formación de profesores en Chile; en algunos casos desde el inicio de los estudios y, en otros, más tardíamente, atendiendo a la necesidad 
de contar con aprendizajes previos al reingreso a la experiencia escolar. Sin embargo, existen al menos dos aspectos compartidos entre todos los casos: (i) la formación práctica se estructura como una línea de formación relevante dentro de los planes de estudio y dotada de una lógica de avance progresivo, ya sea en términos de horas de permanencia en la escuela, complejidad de los focos de estudio o respecto de las oportunidades de intervención directa y asociada a los aprendizajes de los alumnos en el aula; (ii) cada instancia de práctica posee ciertos propósitos específicos, que pueden responder a criterios que van de lo externo a lo interno, de lo simple a lo complejo y desde la descripción y la comprensión a la intervención. En cualquier caso, el aula se constituye en el escenario principal donde se dan a conocer las competencias profesionales de un futuro profesor.

Si bien las primeras instancias de práctica son importantes, por el impacto principalmente emocional que provocan en los futuros profesores, las prácticas de carácter terminal son esenciales para la evaluación y certificación de los aprendizajes y desempeños deseables (perfil de egreso ideal), y de aquellos efectivamente logrados (perfil de egreso real) por un docente ad portas de su graduación y titulación. Focalizar la atención en instancias terminales de práctica permite visibilizar los logros al final de una trayectoria formativa, a la luz de todos los insumos de diseño y monitoreo desplegados para este fin. Así, el logro efectivo de los aprendizajes comprometidos en los perfiles de egreso de las carreras de pedagogía es, de una parte, evidencia fundamental de lo que ha sido la trayectoria de formación realizada por un futuro docente y, de otra, una suerte de promesa de lo que será su desempeño futuro.

Entre los aspectos menos estudiados de este fenómeno se encuentran las múltiples articulaciones que deben producirse para configurar un escenario propicio para la formación docente. A los vínculos entre instituciones debe agregarse articulaciones formativas entre los equipos de gestión de cada escuela y los responsables de la formación universitaria, y, finalmente, las relaciones profesionales y personales entre profesores de las escuelas que reciben en sus 
186 PRÁCTICAS DE ACOMPAÑAMIENTO DE PROFESORES TUTORES EN CARRERAS DE PEDAGOGÍA: ANÁLISIS DE LOS DISPOSITIVOS DE ASEGURAMIENTO Y CERTIFICACIÓN DE LA CALIDAD DE LOS APRENDIZAJES TERMINALES - M. Latorre, C. Vergara, M. Morales, A. Orbeta, C. Escobar, M. Quiroga

aulas a los futuros profesores y tutores que, desde la universidad, acompañan dichos procesos.

Particularmente, es importante profundizar en el rol de los profesores tutores, pues son responsables de "representar" la perspectiva de la formación institucionalizada, considerando los criterios propios de un proyecto académico universitario fundado en argumentos y tradiciones de las ciencias de la educación, y coherente con las demandas de política pública propias de la formación y el ejercicio profesional docente. Más aún, los tutores de prácticas finales son especialmente relevantes en la formación inicial docente, pues constituyen el "último eslabón" de la cadena formativa de la trayectoria de los estudiantes.

En este contexto, resulta relevante abordar las prácticas de acompañamiento que realizan los tutores en instancias de "prácticas finales" de carreras de pedagogía en el campo de la formación inicial de profesores en Chile. Para ello, la pregunta que ha servido de guía al estudio es ¿qué caracteriza las relaciones entre las prácticas de acompañamiento en instancias finales de la formación práctica de carreras de pedagogía y el aseguramiento y certificación de los aprendizajes comprometidos en los perfiles de egreso de tales carreras? A partir de la cual se han formulado otras interrogantes más específicas: ¿quiénes son los profesores tutores de prácticas finales en carreras de pedagogía? ¿Qué características poseen sus prácticas de acompañamiento? ¿Cómo dialoga la formación en instancias de prácticas finales con el aseguramiento y certificación de los aprendizajes comprometidos en los perfiles de egreso de carreras de pedagogía?

A continuación se presenta el marco conceptual que ha servido de base al estudio, luego se describe la metodología empleada y las consideraciones observadas para dar cumplimiento a los estándares éticos involucrados en la investigación en ciencias sociales. Posteriormente se informa de los principales resultados del estudio y se realiza una discusión de estos para, finalmente, formular las conclusiones. 


\section{Marco conceptual}

Chile tiene una larga tradición en formación inicial institucionalizada de profesores, que se remonta a los años cuarenta del siglo XIX, formación que hoy se encuentra tensionada tanto por las demandas de calidad de la formación docente, como por la Nueva Ley de Carrera Docente (ley 20.903). Bajo la lógica de la profesionalización (Martínez Diloné y González Pons, 2010), uno de los componentes clave de esta formación, que pone en jaque a las casas de estudio y al tipo de formación ofrecida, es el de la formación práctica. Sin embargo, las prácticas, como dispositivo de formación, serían un momento privilegiado en la formación inicial, dado que ahí se combinarían una serie de recursos para la futura acción profesional. Así, Correa (2015) las define como un espacio temporal de transición entre formación y profesión, y de actualización de competencias. Rebolledo (2015), siguiendo las ideas de Tejada y Ruiz (2013), agrega que la práctica se comprendería como una

fase compleja que integra diversas dimensiones, conformándose como una interconexión entre el mundo formativo y el productivo, a través del que se desarrollan competencias propias del desempeño profesional, gracias a la implicación en actividades profesionales en contextos y condiciones reales. (p. 140).

Según estos autores, la práctica contrarrestaría la distancia entre la teoría y la práctica.

La formación práctica, en el ámbito universitario y profesoral, remitiría a lo ya expuesto por Lang (1999), a saber, que tendría como finalidad que los futuros docentes construyan y se apropien de aquellas competencias propias de la profesión, como son los saberes prácticos, pero también aquellos de corte teórico. Aquí se revela la tensión existente en la formación de futuros profesores: la relación teoría-práctica o viceversa. Es decir, existiría una dificultad en el proceso de transferir a la práctica los conocimientos teóricos adquiridos en la formación inicial (Cochran-Smith \& Lytle, 1999; Perez-Roux, 2017; Sutherland, Scanlon \& Sperring, 2005), planteándose la necesidad de acortar la distancia entre saberes teóricos 
188 PRÁCTICAS DE ACOMPAÑAMIENTO DE PROFESORES TUTORES EN CARRERAS DE PEDAGOGÍA: ANÁLISIS DE LOS DISPOSITIVOS DE ASEGURAMIENTO Y CERTIFICACIÓN DE LA CALIDAD DE LOS APRENDIZAJES TERMINALES - M. Latorre, C. Vergara, M. Morales, A. Orbeta, C. Escobar, M. Quiroga

y saberes prácticos, mediante la articulación con la realidad escolar desde el inicio de la formación. En este sentido, diferentes autores han llegado al consenso de que la formación inicial docente debe ser un proceso continuo (Feiman Nemser, 2003; Martín-Sospedra, 2015; Mercado Maldonado, 2010; Molina, 2015; Ordóñez y Ocampo, 2005) orientado al desarrollo de competencias, por sobre la reproducción de conocimientos memorísticos.

Por lo demás, hoy existe conocimiento acumulado sobre algunos componentes comunes que deberían tributar a la formación práctica en el ámbito de la docencia o, al menos, tenerlos en consideración (Perrenoud, 2001, 2005; Darling-Hammond, 2001; Korthagen \& Lagerwerf, 2001, 2004; Tardiff, 2004; Hoban, 2006; Russell, 2012, 2014). Se sabe que los futuros profesores traen preconcepciones o saberes experienciales, procedentes de su formación como alumnos, que inciden en sus experiencias de práctica. Por ejemplo, existe evidencia de que los estudiantes que han vivenciado prácticas tradicionales tendrían más probabilidades de identificarse con ellas (Lortie, 1975; Schneuwly, 2002). El problema radica en que estos sujetos observan aspectos superficiales de la enseñanza y no los saberes que los sustentan, llevándolos a una concepción errónea sobre la enseñanza y sus procesos (un error es pensar que enseñar es fácil y, en consecuencia, tienden a imitar los aspectos superficiales de este proceso). Otros autores (Feiman Nemser \& Buchmann, 1989; Richardson, 1996) destacan que algunos estudiantes creen que el aprendizaje es simple y mecánico, es decir, que se centra en transmisión de información de los textos y del profesor a los estudiantes, y que se adquiere a través de escuchar, leer y memorizar. Siguen así un dispositivo de enseñanza de tipo explicación-aplicación, que no concede importancia a la comprensión de los procesos de construcción del conocimiento (Rey, 2001) y que se enmarca en teorías de corte más conductista. Richardson y Placier (2001), por su parte, concluyen que los estudiantes en formación suelen centrarse en aspectos afectivos, en estilos de enseñanza y en estudiantes individuales, no considerando el rol que desempeña el contexto social y el conocimiento pedagógico y disciplinar. Morales y Font (2017) van en la misma línea, indicando que, en el discurso de estudiantes de matemática, al considerar las distintas facetas 
del modelo del conocimiento didáctico-matemático, predominan aquellas de tipo interaccional y afectivo por sobre las epistémicas y cognitivas. Igualmente, los resultados de la investigación de Rodríguez, Armengol y Meneses (2017), focalizada en estudiantes en formación de preescolar y básica, constatan que las competencias más desarrolladas durante las prácticas apuntan al "desarrollo de procesos de interacción y comunicación en el aula, la organización de los alumnos, del tiempo y de los espacios, o el fomento de un clima favorable al aprendizaje y la convivencia" (p. 244).

También se sabe que, para preparar mejor a los futuros docentes, es necesario que ellos pongan en "acción" múltiples intenciones pedagógicas (Kennedy, 1999) mediante experiencias metodológicas diversas, como el estudio de casos y el uso de anécdotas o episodios críticos, entre otras. Al parecer, este tipo de experiencias ayuda a que los estudiantes piensen y actúen de forma profesional al enfrentarse a problemas reales en contextos reales (Hmelo-Silver, 2004). Tejada y Ruiz (2013), al igual que Correa (2011, 2015), relevan modalidades de trabajo en la práctica asociadas a principios socioconstructivistas, como la investigación-acción participativa o colaborativa, y el aprendizaje-servicio. Así aparecen herramientas formativas, como el aprendizaje basado en problemas, el mentoring, el coaching, entre otras.

Diferentes han sido las maneras de enfrentar la formación práctica docente en la enseñanza universitaria. Russell (2014) menciona dos: la "inducción gradual" y la "inducción rápida" a las prácticas. En la primera, la gradualidad estaría dada por el supuesto de que los que están aprendiendo a enseñar requieren de una extensa preparación antes de asumir responsabilidades docentes, y su experiencia como estudiantes no tiene valor. En este tipo de inducción los estudiantes necesitarían primero observar y colaborar en la sala de clases, con el fin de comprender las complejidades del proceso de enseñanza-aprendizaje. Esta inducción, según el autor, "resulta consistente con las tradiciones de una enseñanza y aprendizaje basados en la transmisión: primero el profesor te dice lo que tienes que saber, y luego tienes que ir y ponerlo en práctica" (p. 230). A diferencia de este tipo de inducción, la segunda se fundamenta en 
190 PRÁCTICAS DE ACOMPAÑAMIENTO DE PROFESORES TUTORES EN CARRERAS DE PEDAGOGÍA: ANÁLISIS DE LOS DISPOSITIVOS DE ASEGURAMIENTO Y CERTIFICACIÓN DE LA CALIDAD DE LOS APRENDIZAJES TERMINALES - M. Latorre, C. Vergara, M. Morales, A. Orbeta, C. Escobar, M. Quiroga

que los futuros docentes poseen una vasta experiencia sobre lo que pasa en una sala de clases y, al parecer, aprenderían a ser profesores cuando se les ofrece la oportunidad de realizar clases completas, pues planificarían y pensarían sobre su accionar profesional como lo hace un docente en ejercicio, vivenciando el carácter pluridimensional de las prácticas docentes.

Asimismo, Correa (2015) plantea tres formas en que estos espacios interactúan para formar a los futuros docentes. La primera responde a una concepción aplicacionista de la formación, que consiste en periodos de pasantía en el medio práctico "sin que exista conexión entre las experiencias vividas en la práctica y los contenidos de los cursos que se siguen en la universidad" (p. 263). En esta forma, que suele darse al final de la formación, el establecimiento de práctica es visto como un medio en el que se experimentan las teorías aprendidas en la universidad. La segunda presenta instancias de comunicación entre los dos contextos de formación, es decir, existen instancias en las que teoría y práctica dialogan. "Esta manera (...) es utilizada en aquellos programas que apuntan hacia una mayor integración de los saberes teóricos y prácticos, pero no constituye necesariamente una intención transversal a todo el programa de formación" (p. 264). Finalmente, la tercera modalidad remite a una verdadera articulación y comunicación entre ambos espacios de formación, en la que existen tanto conexiones horizontales como verticales que se nutren mutuamente.

A modo de ejemplo encontramos el caso de Finlandia, en donde la práctica se realiza en el marco de la formación inicial universitaria. Los alumnos tienen periodos de prácticas en centros escolares de forma intermitente a lo largo de todo su proceso de formación, entre cinco a seis semanas al año (Ministry of Education and Culture, 2014, citado en Rebolledo, 2015). En Canadá, en la Universidad de Queen's, se realiza un sistema de alternancia entre la escuela y la universidad desde el primer año, que consiste en que los estudiantes, luego de una semana en la universidad, pasan 16 semanas en la escuela y luego vuelven a un periodo a la universidad (Russell, 2014). En Francia, el segundo año de formación es dedicado a la formación práctica, la que tiene una duración de seis a ocho horas 
semanales en los establecimientos y es supervisada por un tutor. A diferencia de estos países, en Alemania e Inglaterra la formación práctica se ubica en un nivel intermedio entre la formación inicial y la incorporación del profesorado en la escuela, siendo una fase obligatoria que posee una remuneración económica y una duración mínima de un año (Rebolledo, 2015).

Otros países han implementado otras formas de inducción, como las llamadas "prácticas progresivas", en las que el currículo en la universidad se acompaña de actividades prácticas en terreno (Murillo, 2006), que apuntan a solucionar el brusco cambio entre la formación teórica y la inserción de los estudiantes en el espacio laboral, realizado al finalizar su carrera (Avalos \& Aylwin, 2007). Sin embargo, existe evidencia de que muchas de las prácticas tempranas, diseñadas bajo esta forma, son actividades curriculares, con pocas horas semanales, lo que se traduce en pocas horas efectivas en el aula (Russell, 2014). En esta línea, los resultados de algunas investigaciones actuales, como la de Rodríguez et al. (2017), sugieren que las modalidades de formación práctica que combinan periodos intensivos y extensivos de estadía en las escuelas, propiciarían un desarrollo más probable de "competencias asociadas con la aplicación de conocimientos como dominio de conocimientos teóricos sobre la realidad de la escuela y del aula (...), dominio de técnicas y estrategias de seguimiento y control del proceso de enseñanza-aprendizaje" (p. 245). Estos autores insisten igualmente en que, más allá de la modalidad de práctica o de su distribución en los programas de formación, "la identificación de 'centros formadores' adecuados y la consolidación de estructuras de cooperación efectiva entre todos los agentes implicados, constituyen elementos claves" (p. 246) para la formación preprofesional. En nuestro contexto nacional, esto sería un aspecto que podría tensionar y fragilizar la formación, principalmente cuando se hace referencia a la importancia que se le concede a los centros de práctica y a los actores formadores. Martinic et al. (2014) lo destacan en su análisis comparativo de la formación práctica de médicos y profesores en la Pontificia Universidad Católica de Chile: "en Educación no existe un campo de práctica propio de la Facultad y por ello esta descansa en convenios con Establecimientos Educativos seleccionados según criterios de calidad y diversidad de dependencia" (p. 193), destacando 
192 PRÁCTICAS DE ACOMPAÑAMIENTO DE PROFESORES TUTORES EN CARRERAS DE PEDAGOGÍA: ANÁLISIS DE LOS DISPOSITIVOS DE ASEGURAMIENTO Y CERTIFICACIÓN DE LA CALIDAD DE LOS APRENDIZAJES TERMINALES - M. Latorre, C. Vergara, M. Morales, A. Orbeta, C. Escobar, M. Quiroga

también el carácter no contractual y de colaboración por parte de quienes se hacen cargo de esta formación y que evalúan el desempeño de los estudiantes: los docentes de la escuela.

Por lo demás, la literatura concluye que el hecho de tomar distancia permite adaptarse a situaciones inéditas y posibilita aprender a partir de la experiencia (Perrenoud, 2005). Por tanto, para ayudar a los futuros profesores a aprender a pensar sistemáticamente sobre las complejidades que ocurren en la sala de clases (y en los establecimientos), se ha comprobado la necesidad de desarrollar en ellos hábitos metacognitivos que orienten sus decisiones, los que estarían mediados por la reflexión sobre la experiencia práctica personal y la de otros acerca de los múltiples factores que intervienen en el acto de enseñar (Tardif, 2012), la que, a su vez, se estimularía por otros actores: el profesor tutor, un supervisor, un par, un grupo, etc. (Molina, 2014). En consecuencia, modelos como estos han demostrado ser los más exitosos (Darling-Hammond, 1994, 1999; Korthagen, 2005; Tardif, 2012). Sin embargo, este distanciamiento no se evidencia como una tarea fácil para ninguno de los actores implicados en esta formación. Los resultados del estudio de Morales y Font (2017) lo confirman: los estudiantes en práctica no muestran "reflexión profunda de su actividad docente" (p. 134). Las reflexiones solicitadas en los portafolios de práctica no muestran, por ejemplo, el impacto de la formación recibida en los cursos de formación universitaria, siendo estas escuetas y no detallando lo que sucede en el aula, tanto en términos pedagógicos como didácticos.

Tal como enuncian Hirmas y Cortés (2015), actualmente el enfoque crítico-reflexivo habría desplazado al de la racionalidad técnica. El enfoque crítico-reflexivo comprende el proceso educativo como multidimensional, los saberes tienen naturaleza situada y los factores contextuales poseen un peso preponderante. La práctica sería

un proceso complejo en el que no basta con la transmisión de conocimientos, se trata de una actividad crucial en la formación inicial docente, donde los futuros profesores definirán su rol profesional, por lo que debe ser una instancia de reflexión y análisis. (p. 23) 
Apoyándose en las ideas de Labra (2011), las autoras destacan que este componente de la formación debiese reconocer al futuro profesor como un sujeto sociohistórico, con todo lo que ello conlleva. Bajo este marco, la práctica se concibe como un espacio en el que los futuros profesores participan activamente en las distintas dimensiones de desarrollo profesional, en la construcción de conocimientos y como agentes de cambio (Darling Hammond, 1994; Correa, 2014, 2015). Los estudiantes son concebidos entonces como profesionales emergentes, capaces de dar conducción a su propio desarrollo profesional, indagando acerca de su propia enseñanza y, sobre todo, participando en comunidades de aprendizaje.

Si bien la formación práctica parece tomar sentido en el proceso de reflexión que harían los futuros profesores, cabe preguntarse cómo este proceso es guiado y estimulado. Al respecto, el acompañamiento en este campo se comprendería como un "trayecto complejo hacia las profundidades de las propias necesidades, potencialidades y capacidades profesionales, confrontadas por la colectividad de miradas internas y externas que acceden a nuestras prácticas para conocerlas, comprenderlas, apreciarlas y trastocarlas, de modo que puedan orientarse" (Martínez Diloné y González Pons, 2010, p. 9).

El acompañamiento se definiría específicamente como "cualquier dispositivo de apoyo y ayuda implementado, con el fin de permitir que el estudiante se desarrolle profesionalmente, para alcanzar los objetivos previstos y construir las competencias que son propias de la profesión" (Maes, Colognesi \& Van Nieuwenhoven, 2018).

A pesar de que en la literatura actual hay consenso respecto de la conceptualización, lo complejo del acompañamiento aparece al momento de delimitar los actores que lo ejercen, sus destinatarios (futuros profesores de enseñanza básica o media), los tipos de retroalimentación que conlleva o, bien, la forma de organizarlo. Por ejemplo, en cuanto a las modalidades de acompañamiento, Solís, Núñez, Contreras, Rittershaussen, Montecinos y Walker (2011) constataron características de implementación diferentes si la carrera es de educación básica o de educación media. De cierto modo, se 
194 PRÁCTICAS DE ACOMPAÑAMIENTO DE PROFESORES TUTORES EN CARRERAS DE PEDAGOGÍA: ANÁLISIS DE LOS DISPOSITIVOS DE ASEGURAMIENTO Y CERTIFICACIÓN DE LA CALIDAD DE LOS APRENDIZAJES TERMINALES - M. Latorre, C. Vergara, M. Morales, A. Orbeta, C. Escobar, M. Quiroga

observa que las universidades tienden a implementar tres tipos de acompañamiento. Uno conducido solo desde la universidad, otro que focaliza el acompañamiento solo en el establecimiento de práctica y uno de carácter mixto, que combina los dos anteriores.

Respecto al tutor/supervisor, lejos de prevalecer estereotipos como la edad y la experiencia - con los que pudiese preconcebirse la figura de este actor, hoy son múltiples los sujetos que pueden cumplir dicho rol (Ambrosetti, 2010). Según Tejada y Ruiz (2013), el rol del profesor tutor apunta a proporcionar a los actores en formación una experiencia y un tiempo de acomodación preprofesional. Además, el tipo de retroalimentación que se establece entre estos actores y los futuros docentes parece transitar entre dos polos. Por un lado, las retroalimentaciones en las que prevalecen vínculos de tipo afectivo, en las que el tutor se inclina por el reconocimiento y la comprensión; por otro, las que se caracterizan por ser de tipo cognitivo, predominando lo que el futuro docente debería realizar (Mayoral Serrat y Castelló Badia, 2015). Este último tipo de retroalimentación se centraría en la gestión, por parte del sujeto en formación, de lo planificado y lo efectivamente realizado, desde una perspectiva de resolución de problemas con miras a la eficiencia (Maleyrot, 2016). En este tipo de retroalimentación encontramos un amplio espectro, que puede incluir lo disciplinar, lo didáctico, lo pedagógico, lo relacional en la sala de clases, entre otros.

\section{Metodología de la investigación}

Utilizando un enfoque de investigación exploratorio-descriptivo y comprensivo (De Ketele \& Roegiers, 1996), se desarrolla una aproximación teórica y empírica al objeto de estudio y el problema de investigación. El diseño de investigación es cualitativo y considera el estudio de casos múltiples, complementando el uso de estadísticas descriptivas (Savoie-Zajc, 2004) con una comprensión contextualizada del fenómeno, con el fin de lograr una mejor comprensión de este (Yin, 2014).

Para la definición de los casos, se opta por trabajar con dos instituciones universitarias y seleccionar, del total que ofrecen estas 
instituciones, cuatro programas de formación pedagógica de pregrado en ambas y dos adicionales en cada una de ellas, conformando un total de doce casos especificados en la siguiente tabla:

Tabla 1

Programas de formación pedagógica de pregrado seleccionados.

\begin{tabular}{lll}
\hline \multicolumn{1}{c}{ Universidad 1 } & \multicolumn{1}{c}{ Universidades 1 y 2 } & \multicolumn{1}{c}{ Universidad 2 } \\
\hline \multirow{2}{*}{$\begin{array}{l}\text { Pedagogía en Inglés } \\
\text { Pedagogía en Artes Visuales }\end{array}$} & Pedagogía en Lenguaje en Música & Pedagogía en Filosofía \\
& Pedagogía en Matemática & $\begin{array}{l}\text { Pedagogía en Historia } \\
\text { Educación Básica }\end{array}$ \\
\hline
\end{tabular}

Siguiendo el modelo de recogida de información que se presenta en el siguiente diagrama, se desarrollaron diversas instancias que permitieron conformar un corpus de material de investigación constituido por doce planes de estudio, doce perfiles de egreso, doce programas de práctica final, dos entrevistas no estructuradas con responsables de la formación práctica, trece entrevistas a profesores tutores, doce registros de sesiones de prácticas de retroalimentación y una entrevista grupal con estudiantes de práctica final.

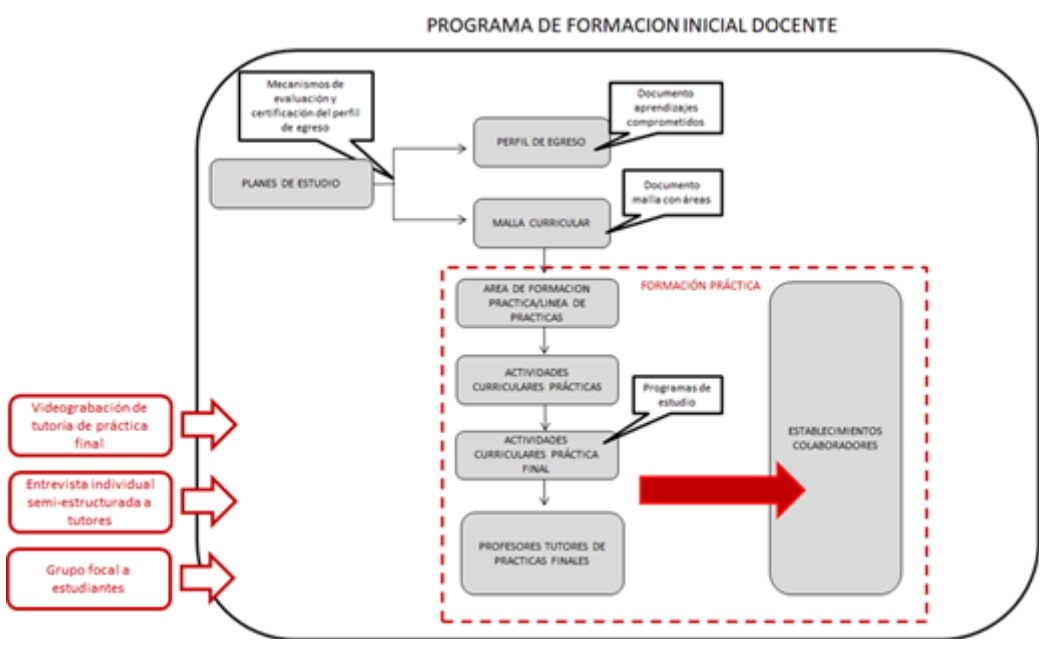

Figura 1. Modelo de recogida de datos. 
196 PRÁCTICAS DE ACOMPAÑAMIENTO DE PROFESORES TUTORES EN CARRERAS DE PEDAGOGÍA: ANÁLISIS DE LOS DISPOSITIVOS DE ASEGURAMIENTO Y CERTIFICACIÓN DE LA CALIDAD DE LOS APRENDIZAJES TERMINALES - M. Latorre, C. Vergara, M. Morales, A. Orbeta, C. Escobar, M. Quiroga

Respecto del análisis de la información, se levantaron categorías temáticas elaboradas a partir de los propósitos que orientaron el estudio, con las cuales se procedió a realizar un análisis cualitativo del material recopilado (Mayring, 2003).

Para el análisis de los planes de estudio y los perfiles de egreso se consideraron como categorías de análisis: (i) la duración del plan de estudio, (ii) el número de actividades formativas totales y el de aquellas correspondientes a la línea de formación práctica, y (iii) las características de los aprendizajes fundamentales comprometidos en los perfiles de egreso, relevando la naturaleza de los aprendizajes, el enfoque y orientación de la formulación, y el foco de estos.

En el análisis de las entrevistas y el registro videograbado de prácticas de acompañamiento se utilizaron las siguientes categorías de análisis (tabla 2): 
Tabla 2

Categorías para análisis de entrevistas a tutores, focus a estudiantes y prácticas de acompañamiento.

\begin{tabular}{|c|c|c|c|}
\hline Categorías temáticas & $\begin{array}{c}\text { Entrevistas a tutores de } \\
\text { prácticas }\end{array}$ & Focus a estudiantes & $\begin{array}{c}\text { Prácticas de } \\
\text { acompañamiento }\end{array}$ \\
\hline $\begin{array}{l}\text { Aprendizajes } \\
\text { comprometidos en } \\
\text { los perfiles de egreso } \\
\text { y los dispositivos } \\
\text { de evaluación y } \\
\text { certificación de } \\
\text { aprendizajes terminales } \\
\text { de carreras de } \\
\text { pedagogía }\end{array}$ & $\begin{array}{l}\text { 1. Características de } \\
\text { la línea de prácticas } \\
\text { de la carrera } \\
\text { 2. Cursos de la malla } \\
\text { curricular de la } \\
\text { carrera que tienen } \\
\text { un vínculo directo } \\
\text { con la práctica final } \\
\text { 3. Aprendizajes } \\
\text { terminales } \\
\text { esperados de la } \\
\text { práctica final } \\
\text { 4. Evaluación del } \\
\text { logro de estos } \\
\text { aprendizajes }\end{array}$ & $\begin{array}{l}\text { 1. Características } \\
\text { que tiene la línea } \\
\text { de prácticas de la } \\
\text { carrera que cursas } \\
\text { 2. Cursos de la malla } \\
\text { curricular de la } \\
\text { carrera que tienen } \\
\text { un vínculo directo } \\
\text { con la práctica final } \\
\text { 3. Aprendizajes que } \\
\text { se espera logren } \\
\text { los estudiantes } \\
\text { al término de la } \\
\text { práctica final } \\
\text { Evaluación del } \\
\text { logro de estos } \\
\text { aprendizajes }\end{array}$ & $\begin{array}{l}\text { 1. Aprendizajes } \\
\text { explícitos } \\
\text { presentes }\end{array}$ \\
\hline
\end{tabular}

Características socioprofesionales de los profesores tutores que acompañan a estudiantes en instancias de prácticas finales de carreras de pedagogía
5. Condiciones en que se desempeñan los tutores de práctica final

6. Principales tareas y funciones de un tutor/a de práctica final

7. Procesos de selección de los tutores que acompañan el proceso de práctica final

8. Formación y/o especialización específica de los tutores de las distintas instancias de prácticas

Prácticas de acompañamiento que realizan los profesores tutores en instancias de prácticas finales de carreras de pedagogía, con foco en los aprendizajes comprometidos y las modalidades de evaluación de dichos aprendizajes
9. Características de las acciones de acompañamiento de tutores a estudiantes en la práctica profesional/ final

10. Características de la retroalimentación de tutores a estudiantes
5. Principales tareas y funciones de un tutor/a de práctica final
6. Características de acciones de acompañamiento que realizan los tutores a estudiantes en la práctica profesional/final: tipo, modalidad, frecuencia.

7. Características de la retroalimentación de tutores a estudiantes en la práctica final: tipo, modalidad, frecuencia.
2. Foco de atención

3. Actores involucrados en la instancia

4. Acciones de retroalimentación que realiza el tutor hacia el estudiante

5. Contexto en el cual se realiza la instancia

6. Tiempo de duración 
198 PRÁCTICAS DE ACOMPAÑAMIENTO DE PROFESORES TUTORES EN CARRERAS DE PEDAGOGÍA: ANÁLISIS DE LOS DISPOSITIVOS DE ASEGURAMIENTO Y CERTIFICACIÓN DE LA CALIDAD DE LOS APRENDIZAJES TERMINALES - M. Latorre, C. Vergara, M. Morales, A. Orbeta, C. Escobar, M. Quiroga

\begin{tabular}{|c|c|c|c|c|}
\hline $\begin{array}{l}\text { Relaciones entre } \\
\text { las características } \\
\text { de las prácticas de } \\
\text { acompañamiento } \\
\text { que realizan los } \\
\text { profesores tutores, } \\
\text { los aprendizajes } \\
\text { comprometidos en } \\
\text { los perfiles de egreso } \\
\text { y su aporte a la } \\
\text { configuración de la } \\
\text { identidad profesional } \\
\text { de docentes noveles }\end{array}$ & $\begin{array}{l}\text { 11. Relaciones entre las } \\
\text { prácticas finales, } \\
\text { los aprendizajes } \\
\text { del perfil de egreso } \\
\text { y la conformación } \\
\text { de identidad } \\
\text { profesional } \\
\text { de docentes } \\
\text { principiantes } \\
\text { 12. Mejoras posibles } \\
\text { de incorporar al } \\
\text { acompañamiento en } \\
\text { las prácticas finales }\end{array}$ & 9. & $\begin{array}{l}\text { Relaciones entre las } \\
\text { prácticas finales, } \\
\text { los aprendizajes } \\
\text { del perfil de egreso } \\
\text { y la conformación } \\
\text { de identidad } \\
\text { profesional } \\
\text { de docentes } \\
\text { principiantes } \\
\text { Sugerencias para el } \\
\text { mejoramiento del } \\
\text { acompañamiento } \\
\text { de tutores en las } \\
\text { prácticas finales }\end{array}$ & $\begin{array}{l}\text { 7. Relaciones } \\
\text { explícitas a las } \\
\text { que alude en } \\
\text { la instancia, } \\
\text { respecto de las } \\
\text { relaciones entre } \\
\text { aprendizajes } \\
\text { involucrados y } \\
\text { configuración } \\
\text { de la identidad } \\
\text { y rol del futuro } \\
\text { docente }\end{array}$ \\
\hline
\end{tabular}

Respecto del cumplimiento de los estándares éticos correspondientes a la investigación en ciencias sociales y de acuerdo con las orientaciones vigentes para el trabajo con seres humanos, se solicitó el pronunciamiento del Comité de Ética de la institución principal acerca del estudio y de las instancias de trabajo directo con personas, así como la aprobación, por parte del mismo comité, de las cartas de consentimiento informado que leyó y firmó cada uno de los participantes.

\section{Resultados de la investigación}

a. El lugar de la formación práctica en los planes de estudio de las carreras de pedagogía 
CALIDAD EN LA EDUCACIÓN nº 53, diciembre 2020199

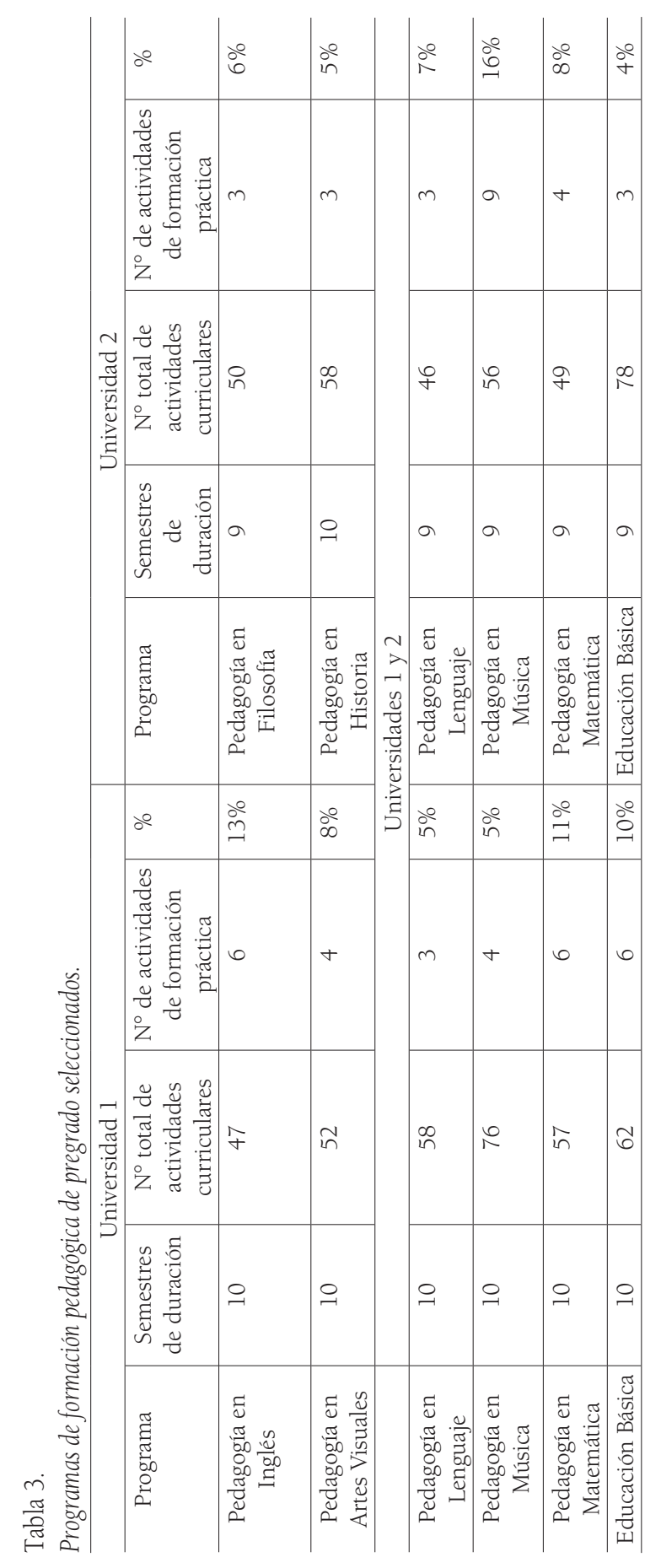


200 PRÁCTICAS DE ACOMPAÑAMIENTO DE PROFESORES TUTORES EN CARRERAS DE PEDAGOGÍA: ANÁLISIS DE LOS DISPOSITIVOS DE ASEGURAMIENTO Y CERTIFICACIÓN DE LA CALIDAD DE LOS APRENDIZAJES TERMINALES - M. Latorre, C. Vergara, M. Morales, A. Orbeta, C. Escobar, M. Quiroga

Como se muestra en la tabla 3, los currículos de formación de profesores tienen duraciones diferentes, los planes de estudio están sobrecargados y la formación práctica tiene una presencia limitada.

Del total de programas analizados, todos los programas de la institución 1 poseen diez semestres de duración; mientras que en la institución 2 solo uno posee diez semestres de formación y todos los demás nueve semestres.

La recarga en los planes de estudio se nota especialmente en los programas de nueve semestres, pues tienen un elevado número de actividades de formación por periodo, entre seis y nueve; incluso, hay programas que consideran nueve actividades de formación a realizar en paralelo, sumándose una alta dispersión de las actividades formativas, las que van desde 46 a 76 . Sin embargo, si se considera el promedio de actividades totales de cada programa por institución, se obtiene que para la institución 1 el promedio es 59, mientras que para la 2 es de 56.

Las actividades de formación práctica no superan, en promedio, el $10 \%$ del total de las actividades de formación en una carrera de pedagogía. En la institución 1 el número de instancias de formación práctica va entre tres y seis, con promedio de este último número. En el caso de la institución 2 el número de prácticas va entre tres y nueve, con promedio de este último dato.

b. Los aprendizajes esperados de la formación práctica hacia el término de la formación inicial docente

En los perfiles de egreso de las carreras estudiadas no se advierte explícitamente una formulación compartida a nivel institucional. La mayoría de los casos comparte una estructuración basada en dos componentes: el primero, un párrafo inicial que describe algún rasgo del futuro docente ligado a su rol socioprofesional. A modo de ejemplo se señala:

... especialista con dominio avanzado de la (disciplina), tanto en forma oral como escrita, que tiene conocimiento experto de la metodología para enseñarla en la sala de clases y que necesita enriquecerse de 
múltiples perspectivas para el desarrollo de su pensamiento. (Carrera $1-\mathrm{A})$

... un profesional formado en el marco del humanismo cristiano, que posee una formación que manifiesta en competencias disciplinarias, pedagógicas y prácticas integradas... (Carrera 2-A)

En algunos casos, pertenecientes a la institución 2, esta formulación inicial refiere directamente a cualidades que se espera de su docencia. Por ejemplo:

... el titulado ejerce la docencia atendiendo con interés el conocimiento del grupo destinatario de la formación, potencia un tipo de aprendizaje interactivo, que permite mediar y facilitar dicho aprendizaje en sus alumnos. Asume las situaciones inesperadas y las de difícil control... puede ejercer su labor docente con capacidad de análisis, espiritu crítico $y$ de diálogo con los pensadores relevantes, y puede asumir libremente la visión cristiana. (Carrera 2- C)

A diferencia de los casos anteriores, se observa, en otros perfiles de egreso, que se ha seguido la estructura del "Marco para la buena enseñanza"; por último, hay perfiles de egreso que señalan ejes inspiradores.

El segundo componente de los perfiles de egreso se refiere a los aprendizajes específicos que lo componen. En la institución 1 se consideran ciertos objetivos o ámbitos (pedagógico, disciplinar, cultural, valórico, específico), mientras que en la institución 2 se estructuran sobre la base de tres tipos de competencias: genéricas, disciplinares y profesionales; las subcompetencias que conforman cada grupo son numerosas, pudiendo alcanzar hasta treinta enunciados en un mismo perfil.

Sobre la presencia de aprendizajes ligados a la práctica final, se constata principalmente capacidades ligadas a la planificación, el diseño y la implementación de estrategias de enseñanza para el logro de aprendizajes en la escuela; se incorporan competencias para formular metas y secuencias de aprendizaje, y seleccionar recursos didácticos en el marco del proyecto educativo del establecimiento y 
202 PRÁCTICAS DE ACOMPAÑAMIENTO DE PROFESORES TUTORES EN CARRERAS DE PEDAGOGÍA: ANÁLISIS DE LOS DISPOSITIVOS DE ASEGURAMIENTO Y CERTIFICACIÓN DE LA CALIDAD DE LOS APRENDIZAJES TERMINALES - M. Latorre, C. Vergara, M. Morales, A. Orbeta, C. Escobar, M. Quiroga

el marco curricular nacional. Ligada a las situaciones de enseñanza está la contextualización de éstas.

Llama la atención que las estrategias de evaluación de los aprendizajes aparezcan como un foco de atención con menor presencia que otros elementos y en varios perfiles no están contenidas.

\section{c. Características de los tutores que acompañan las prácticas finales}

En la institución 1 los tutores, en su mayoría, poseen contrato a honorarios y la dedicación es flexible. Los entrevistados señalan inconsistencias entre los tiempos que dedican a la tutoría y los tiempos para los cuales están contratados. Se declara, además, que requieren adaptarse a los horarios de los estudiantes para realizar las tutorías, que consideran como mínimo dos visitas a la escuela, cada 15 días y con una duración de una hora con cada estudiante.

Las principales tareas de los tutores de la institución 1 se relacionan con vincular la teoría, la experiencia y la práctica; realizar acompañamiento, evaluar, retroalimentar individual o grupalmente al estudiante permanentemente, además de entregarle herramientas nuevas cuando aparecen temas emergentes; apoyar en los contenidos disciplinares; coordinar acciones con la escuela y reunirse con la coordinadora de prácticas de la carrera que trabaja en la universidad.

En la institución 2 se explicita que el tutor de práctica final debe cumplir 22 horas de dedicación, tiempo que distribuye entre las visitas a las aulas de práctica en los distintos establecimientos a los que asisten los futuros docentes, las tríadas en la interacción con los profesores mentores, las tutorías, los talleres, además de la corrección de trabajos y reuniones de tutores y de carrera. El número de estudiantes fluctúa entre cinco y doce por tutor. Las visitas son mínimo dos, pero por lo general una vez al mes, a lo que se agrega talleres de 90 minutos cada 15 días. Un aspecto característico de la segunda institución son las reuniones con las tríadas (profesor de la universidad, profesor del establecimiento y estudiante en práctica), que se suman a reuniones semanales y talleres, ante lo cual los tutores destacan la sobrecarga de obligaciones y la escasez de tiempo. 
Las principales funciones del tutor en la institución 2 consisten en apoyo en lo disciplinar, lo pedagógico y lo personal. El tutor es, además, el acompañante pedagógico desde la universidad, interlocutor y representante de la institución universitaria en la escuela, y mediador con el colegio, en tanto centro de práctica, y con el mentor específico de cada estudiante.

Si bien en ambas instituciones los tutores reconocen en la experiencia profesional y personal la principal base de su participación en este rol, declaran que en ninguna existe un programa de formación específico, aunque sí identifican algunas instancias valiosas que tampoco son obligatorias.

\section{d. Perspectiva de los tutores respecto de la práctica final}

En la universidad 1 se señala que en la línea de práctica existe una perspectiva o enfoque compartido y conocido, que va desde lo pedagógico, lo profesional y lo disciplinar a lo didáctico, que sería lo más complejo; desde el contexto hasta llegar al aula, la planificación y el desarrollo de las clases. Se menciona que hay un trabajo de vinculación con la escuela y con los profesores guías, y un acompañamiento a los estudiantes en la preparación de sus clases y en los aspectos personales que involucra la práctica. Al respecto, algunos tutores indican:

.. la práctica va en una línea progresiva, que avanza desde un acercamiento a la escuela, al centro escolar, desde una mirada y análisis desde la cultura, desde la forma de organización que tiene la escuela, hasta quinto año que ya es la especialización y práctica en una didáctica determinada.

En la universidad 2 cada actividad de formación práctica posee un enfoque distintivo, con niveles diversos de exigencia o distintos niveles de habilidades, aunque declaran una línea común de reflexión e investigación docente, que camina hacia la especialización didáctica y responsabilidad profesional. Por ejemplo, un tutor señala:

... en la práctica inicial, el trabajo de tutoría está en manos de la escuela de pedagogía, porque se centró y se enfocó en el tema de los 
204 PRÁCTICAS DE ACOMPAÑAMIENTO DE PROFESORES TUTORES EN CARRERAS DE PEDAGOGÍA: ANÁLISIS DE LOS DISPOSITIVOS DE ASEGURAMIENTO Y CERTIFICACIÓN DE LA CALIDAD DE LOS APRENDIZAJES TERMINALES - M. Latorre, C. Vergara, M. Morales, A. Orbeta, C. Escobar, M. Quiroga

aprendizajes. En la intermedia, nosotros tenemos una fuerte presencia, y en la final, porque la intermedia está concentrado con didáctica...

Respecto de las asignaturas que se vinculan directamente con la práctica, el discurso de los tutores revela que son las disciplinares, las didácticas y las de reflexión. En la institución 1 el principal nexo está en los talleres de titulación y reflexión; mientras que en la institución 2 se reconoce un mayor anclaje en aquellas de tipo didáctico.

Los principales aprendizajes esperados al término de la práctica final se relacionan con capacidades o competencias específicas. En la institución 1 se trata de capacidades ligadas a la enseñanza de la disciplina y a la formación ética que debe tener un profesor, con sello de la institución formadora: proactividad, autonomía, responsabilidad, conocimiento del contexto, dominio didáctico y un alto nivel de reflexión de la propia práctica. La institución 2 se centra en capacidades de orden pedagógico, disciplinar y didáctico, destacando la reflexión docente sobre la contingencia, pensamiento pedagógico flexible, manejo disciplinar, pertinencia de las estrategias de enseñanza seleccionadas, integración al contexto escolar, realizar prácticas efectivas e innovar en la enseñanza con el uso de recursos y metodologías diversas.

En ambas instituciones, la evaluación de la práctica final se basa en una producción escrita, la cual, si bien considera recorridos distintos, tiene como producto final un informe que contiene: marco teórico, secuencia didáctica y reflexiones del estudiante, para el caso de la institución 1. Y, para el caso de la institución 2, un documento que incluye: diagnóstico, planificación, análisis de la práctica y autoevaluación.

Respecto del acompañamiento en la práctica final, lo primero que llama la atención es que, en ambos casos, se habla de un acompañamiento individual y con alta presencialidad. Sin embargo, el discurso de los actores evidencia diferencias. En la institución 1 se indica que no está muy definido dentro de las carreras y que el acompañamiento correspondería a las observaciones de clases, la retroalimentación grupal y/o individual, a reportes o mini informes 
de cada una de las clases y a la evaluación que hace el tutor. Se considera también la presencia de algunos elementos teóricos, para que los estudiantes tengan más herramientas y puedan discernir, reflexionar, analizar, comparar su propio desempeño práctico y lo que están vivenciando en los centros de práctica. El principal modelo o idea en función es la metacognición. Ejemplos del discurso de los tutores son los siguientes:

... les hacemos hacer algunos trabajos escritos como de análisis. Ellos analizan, por ejemplo, videos de clase de sus compañeros, después analizan un video de clase de ellos. Nosotros vamos a observar también las clases.

... va evaluando a través de pautas de evaluación que vamos haciendo por clase, entonces, si tú tomas todas las pautas de un estudiante a final de año ves los progresos.

En la institución 2, en cambio, se menciona que el acompañamiento está normado por protocolos; que se trata de un acompañamiento de tipo pedagógico y de carácter presencial, con disposición permanente para el estudiante. Algunos tutores señalan:

... tenemos una unidad de práctica que tiene ciertas rúbricas sistematizadas que te permiten protocolizar el proceso.

... junto con la conversación que tenemos con el profesor mentor, las tríadas y la observación de clases, hacemos la apreciación evaluativa en base, digamos, a los protocolos que entrega la universidad.

La retroalimentación a los estudiantes es variada en cuanto a modalidades e instancias. Mientras que la institución 1 declara que la retroalimentación está asociada y hace parte de la evaluación, aunque también se realiza en los talleres, la institución 2 expresa que esta se lleva a cabo en el marco de talleres y tutorías. En la institución 1 hay retroalimentaciones individuales y grupales y, por lo general, no consideran una pauta. En la institución 2 las retroalimentaciones se llevan a cabo además con el mentor (profesor(a) de la escuela) en el marco de las tríadas, pero son individuales y en algunas carreras son guiadas por una pauta y con preguntas adicionales. 
206 PRÁCTICAS DE ACOMPAÑAMIENTO DE PROFESORES TUTORES EN CARRERAS DE PEDAGOGÍA: ANÁLISIS DE LOS DISPOSITIVOS DE ASEGURAMIENTO Y CERTIFICACIÓN DE LA CALIDAD DE LOS APRENDIZAJES TERMINALES - M. Latorre, C. Vergara, M. Morales, A. Orbeta, C. Escobar, M. Quiroga

Los tutores de ambas instituciones coinciden en señalar que la práctica final es una instancia de síntesis de la formación. La institución 1 destaca que se hace un trabajo con el perfil de egreso, a través de un ejercicio de análisis de la enseñanza que realiza el futuro docente, con foco en los logros alcanzados en la práctica y los propuestos en el perfil. Sin embargo, dicho perfil queda implícito y tampoco es evidente su forma de verificación.

En la institución 2, la práctica final aparece, según los tutores, asociada a la malla. Ésta está pensada desde las didácticas y desde los espacios de reflexión, permitiendo comprobar si van logrando el perfil de egreso, pero además permite evaluar la integración a la comunidad educativa, porque los profesores en formación tienen que poner en práctica todos los contenidos o todos los lineamientos que tiene este perfil, que remiten al componente pedagógico, al componente disciplinar y a la parte valórica de la institución.

\section{e. La perspectiva de los estudiantes respecto de la práctica final}

En el relato de estudiantes que se encuentran desarrollando su práctica profesional, es posible identificar elementos comunes y diferenciadores entre carreras de una misma universidad. Es así como identifican la actividad curricular "didáctica", en cada una de las disciplinas, como una actividad que se relaciona directamente con la práctica. Tres carreras de media reconocen también un aporte a algunas actividades de la línea pedagógica y, en particular, una de ellas le otorga gran importancia a la actividad de evaluación.

Ninguno de los estudiantes de las carreras identifica o relaciona alguna actividad del ámbito disciplinar asociada a la práctica.

En términos de los aprendizajes logrados durante las instancias de prácticas, los estudiantes relevan aspectos que no están declarados específicamente en los programas de estas actividades curriculares. Entre los aspectos señalados destaca el reconocimiento de que

... la pedagogía no es para todos. 
... ser profe no es para cualquiera.

En palabras de los estudiantes, el hecho de estar inserto en un espacio educativo les permite reconocer aspectos importantes de la identidad profesional y agregan que

... la disciplina es una excusa para enseñar cosas mayores.

Además, reconocen como un aspecto importante y que no se entrega en la universidad, el desarrollo de una inteligencia emocional. También destacan haber aprendido aspectos burocráticos de la escuela, lo cual, según ellos, no se enseña en las instancias de formación o preparación para las prácticas o el taller de reflexión. La escuela tiene protocolos que deben ser aprendidos en las prácticas, pero no hay un espacio de reflexión al respecto.

A propósito del rol de los tutores, las percepciones son muy diversas. Mientras en una carrera se señala que el tutor tiene alrededor de diez estudiantes a cargo, otras señalan que tienen alrededor de cinco, y otra de dos a tres estudiantes.

Respecto del número de visitas al centro de práctica, no hay grandes diferencias. La mayoría observa dos clases y, además, reconocen que los tutores van a presentarlos (o a presentarse) al establecimiento y, hacia el término del periodo, hacen un cierre. Es decir, podemos señalar que, en general, los tutores van cuatro veces por estudiante al centro de práctica.

Acerca de la dedicación de los tutores en los procesos de acompañamiento, evaluación y retroalimentación, las percepciones son variadas. Algunos reconocen que los tiempos son limitados, que los tutores solo van a observar la clase y se retiran; en cambio, otros expresan que los tutores hacen un acompañamiento más extenso, en tiempos fuera del horario asignado, pues van a observar y luego, en el mismo establecimiento, hacen una retroalimentación. También reconocen que hay una limitante relativa al tipo de tutor que tienen: cuando el tutor es un profesor de planta de la universidad hay mayor posibilidad de acceder a consultas y ayudas; en cambio, cuando el tutor es un "profesor hora" los tiempos y espacios son limitados. 
208 PRÁCTICAS DE ACOMPAÑAMIENTO DE PROFESORES TUTORES EN CARRERAS DE PEDAGOGÍA: ANÁLISIS DE LOS DISPOSITIVOS DE ASEGURAMIENTO Y CERTIFICACIÓN DE LA CALIDAD DE LOS APRENDIZAJES TERMINALES - M. Latorre, C. Vergara, M. Morales, A. Orbeta, C. Escobar, M. Quiroga

Finalmente, los estudiantes perciben como una gran debilidad que la universidad no trabaje desde los espacios colaborativos. Piensan que contribuiría a su formación si hubiera instancias comunes a las diferentes carreras, en la lógica de que todas son carreras de pedagogía y que, luego, cuando trabajen en la escuela, deberán trabajar en equipo con los otros profesores.

\section{f. Características del acompañamiento y retroalimentaciones de tutores a estudiantes en instancias de práctica final}

A partir del análisis de los espacios de acompañamiento de los estudiantes de pedagogía que se encuentran desarrollando su práctica profesional, se observa que solo una carrera realizó la retroalimentación en el establecimiento educacional con la compañía de las profesoras guías. El resto de las carreras observadas efectuaron la retroalimentación en espacios universitarios.

La dinámica de retroalimentación que se observó, muestra que la carrera A desarrolla esta actividad en el colegio e involucra la participación de los profesores guías; en cambio, en la carrera C la retroalimentación se hace de manera colectiva y participan dos tutores y ocho estudiantes. El resto de las carreras desarrolla este proceso de manera individual, es decir, un profesor tutor y un estudiante.

No hay un criterio común respecto del tiempo de duración de la retroalimentación, variando entre 21 minutos, la más corta, y 96 minutos, la más extensa.

Al observar los contenidos trabajados en las retroalimentaciones se identifican tres momentos, con matices en las diferentes carreras, sin un elemento unificador por universidad.

Momento 1. Al inicio de la retroalimentación: en el caso de la carrera $C$, que correspondió a la retroalimentación grupal, este espacio partió tratando otros aspectos no asociados directamente con la práctica, como son procesos de cierre, tiempos para el examen, en el marco de orientar y normalizar esta instancia evaluativa. La carrera A, que desarrolló su retroalimentación en el espacio educativo, inició la sesión de retroalimentación con la evaluación de las profesoras guías 
(esta estudiante tenía dos profesores guías en la escuela). En el caso de las carreras B, D y $G$ la retroalimentación se inició preguntando cómo se sintieron realizando su clase. Para la carrera F, en cambio, se inició con un registro de la hora de inicio de la actividad y especificando qué aspectos se trabajarían en esa sesión. Finalmente, la carrera E inició la retroalimentación entregando inmediatamente una evaluación de la clase desarrollada por el estudiante.

Momento 2. En la parte más sustantiva de la retroalimentación, la mayoría de los tutores hace una apreciación general de lo desarrollado en la clase y luego analizan situaciones específicas. Esto no es posible visualizarlo en las carreras A y $C$, pues estas son las que hacen la tríada y la retroalimentación grupal, respectivamente. En el caso de la carrera A, lo importante en esta etapa es que se les consulta a los profesores guías respecto de cómo la escuela resuelve ciertos temas, como por ejemplo cuando deben clases en la tarde. Los profesores guías dedican tiempo a compartir su experiencia como profesores en dicho establecimiento, pero esto no necesariamente se relaciona con la experiencia de la estudiante en práctica o con una situación puntual que hubo que resolver en la clase desarrollada por ella. En la carrera C, al ser una retroalimentación general, las temáticas planteadas también son generales: los estudiantes exponen diferentes situaciones que les suceden en el establecimiento o en su práctica específica, una suerte de puesta en común de estas situaciones y una reflexión por parte de los tutores respecto de lo acertado de la acción o de lo que ellos harían en ese caso. La reflexión es, desde lo genérico, acerca de diversos objetos de atención.

Momento 3. Fase de cierre, en la cual, en general, se entrega una mirada positiva al estudiante en práctica, motivándolo para lo que viene, deseándoles éxito y parabienes. En el caso de la carrera $A$, el foco está en el agradecimiento para con las profesoras guías, invitándolas a seguir trabajando con la universidad y a concurrir a las actividades de finalización.

En términos del trabajo realizado con los estudiantes, se evidencia que la carrera $C$ no trabaja con el apoyo de algún registro escrito. En este caso, el trabajo de reflexión que se desarrolla está 
210 PRÁCTICAS DE ACOMPAÑAMIENTO DE PROFESORES TUTORES EN CARRERAS DE PEDAGOGÍA: ANÁLISIS DE LOS DISPOSITIVOS DE ASEGURAMIENTO Y CERTIFICACIÓN DE LA CALIDAD DE LOS APRENDIZAJES TERMINALES - M. Latorre, C. Vergara, M. Morales, A. Orbeta, C. Escobar, M. Quiroga

en torno a lo que plantean los estudiantes como experiencias o situaciones generales y/o específicas. Ante esto, los tutores modelan posibles salidas a dichas situaciones, posicionados desde la propia experiencia. Y, si bien es una retroalimentación grupal, los tutores velan porque todos los estudiantes intervengan con alguna apreciación o comentario. En la carrera A el tutor mantiene un documento escrito, el cual parece ser una pauta, que mira durante la conversación sin hacer alusión directa a algún punto de esta. El foco de lo trabajado parte desde la retroalimentación dada por uno de los profesores guías, quien tiene un registro en un cuaderno con el punteo de los temas. En el caso de las carreras B y D los profesores tutores trabajan en función de anotaciones o del registro de un cuaderno. Este tiene elementos de la observación de la práctica que orientan la retroalimentación. Se pudo apreciar que este tipo de registro contiene elementos que llamaron la atención del tutor y sobre los cuales intenciona lo que deben mejorar los estudiantes. En las carreras E y $G$ los tutores trabajan en función de una pauta que contiene distintos puntos y extensiones. Por lo que se logra observar en la retroalimentación, esta pauta fue completada por ellos en las visitas a los centros de prácticas. En dinámicas parecidas, los tutores refuerzan elementos logrados e indican las debilidades observadas. Por último, en el caso de la carrera $F$ se aprecia que mantiene ambos insumos - un cuaderno con un registro y una pauta-, ambos elementos completados durante una visita previa al centro de práctica. Algo que se observa transversalmente es que los tutores hablan a los estudiantes de la misma situación en otro espacio, con otras características, en la lógica de "...cuando te toque tal situación, tú deberías...".

\section{Discusión de resultados y conclusiones del estudio}

En primer lugar, a modo de contexto, el lugar que ocupa la formación práctica en las carreras que conformaron la muestra, en comparación con las otras actividades de formación, como los cursos por ejemplo, no es relevante. Los resultados mostraron que esta formación ocupa en promedio solo el 10\% del total de actividades de formación que conforman los planes de estudio en las carreras analizadas. Es importante señalar que este dato no consideró las horas ni los créditos implicados en estas actividades formativas, debido a que dicha información no 
siempre está disponible y, de estarlo, no es fácilmente comparable. Sin embargo, los resultados del estudio dejan constancia de que la mayor parte de la formación de los futuros docentes se realizaría en las universidades, enmarcándose en lo que Russell (2014) llama "prácticas de inducción gradual". Así, en las prácticas analizadas, los estudiantes, a pesar de iniciar su formación práctica antes de su último año de formación, requerirían de conocimientos formales antes de iniciarse en la profesión, con el objeto de comprender las complejidades de la docencia. Ellos primero observan clases, las analizan y, luego, al final, pueden realizarlas. Además, las actividades de formación práctica corresponden a instancias específicas ubicadas en periodos claves del recorrido formativo, no conformando un proceso continuo, como sería deseable, de acuerdo con los planteamientos de Feiman Nemser, 2003; Martín-Sospedra, 2015; Mercado Maldonado, 2010; Molina, 2015; Ordóñez y Ocampo, 2005, entre otros.

En segundo lugar, los resultados mostraron que, en su mayoría, los profesores acompañantes de estas prácticas no son parte de las plantas docentes de las universidades y las tareas que cumplen son múltiples, variadas y, según su discurso, exceden al tiempo de contrato. Además, no tienen formación específica en su rol, prevaleciendo su experiencia. No existen premisas comunes en cuanto a las tareas a desarrollar por estos sujetos acompañantes, las que transitan entre la observación de las prácticas de los estudiantes en el aula y ser los representantes de las casas de estudios en los centros de práctica. Además, se encontraron otras tareas de índole diversa: hacer retroalimentación sobre lo realizado y hacerse cargo de los aspectos tanto cognitivos como personales/emocionales que conlleva la estadía en la escuela. Así, los resultados dan cuenta de que el rol del profesor o profesora acompañante de la formación práctica es, en términos contractuales, periférico, definido vagamente y con tareas variadas, lo que en cierto modo deja la responsabilidad del desempeño en manos y según criterios de los propios tutores.

En tercer lugar, se constatan enfoques distintos de cómo enfrentar la práctica final, pero que convergen en el quehacer docente, específicamente en la enseñanza de una disciplina en particular. Nuevamente aparece la lógica de las prácticas progresivas, partiendo 
212 PRÁCTICAS DE ACOMPAÑAMIENTO DE PROFESORES TUTORES EN CARRERAS DE PEDAGOGÍA: ANÁLISIS DE LOS DISPOSITIVOS DE ASEGURAMIENTO Y CERTIFICACIÓN DE LA CALIDAD DE LOS APRENDIZAJES TERMINALES - M. Latorre, C. Vergara, M. Morales, A. Orbeta, C. Escobar, M. Quiroga

con conocimientos pedagógicos, luego con los disciplinares, seguidos de los didácticos para finalmente llegar a lo que el estudiante debe hacer en el aula. Algo similar ocurre con lo que ellos esperan que los estudiantes aprendan en esta práctica final: el análisis de aspectos más bien descriptivos y no sustantivos de la enseñanza y el aprendizaje es lo común. La diferencia aparece en la declaración de las capacidades enunciadas en los perfiles de egreso de las carreras de la muestra. En suma, la práctica, en tanto objeto de análisis, se muestra parcelada: el saber docente no se trata como tal y la multidimensionalidad de la práctica docente suele comprenderse como la agregación de un todo desarticulado.

En cuarto lugar, el acompañamiento que hacen los tutores deja en evidencia que la presencialidad es lo principal y no se distinguen modelos definidos y consensuados. Las diferencias se presentan incluso al interior de una misma carrera. El discurso solo confirma la existencia de una alta presencialidad. Existen diferencias en las modalidades, se encuentran instancias que se llevan a cabo de forma individual y grupal; en otras, se incluye desde la visita a la escuela hasta la reunión con el estudiante en práctica, sin hacer referencia a un dispositivo de ayuda profesional, cualquiera sea, que permita el futuro desarrollo profesional del estudiante, como lo mencionan Maes et al. (2018). Entonces, el acompañamiento queda definido solo cuando existen pautas o protocolos, dando a entender que la práctica docente en sí correspondería a acciones previstas y diseñadas con antelación, predominando una relación de causalidad lineal que busca, en consecuencia, la aplicación eficaz de estrategias producidas fuera de la escuela y que conformarían el quehacer docente de los futuros profesionales.

En relación con lo anterior, se verifica que las prácticas de acompañamiento poseen modalidades organizativas distintas, que no dan cuenta de patrones definidos ni siquiera al interior de una misma carrera; algo similar, pero a nivel micro, a lo encontrado en el estudio de Solís et al. (2011). Solo se comprueba que, en todos los casos, existe retroalimentación y se realizan reflexiones. En cuanto a las retroalimentaciones, se conformarían en general al menos en tres momentos: uno inicial, uno intermedio y uno de cierre. El momento que se muestra más delimitado es el intermedio, y es aquel en el que 
los tutores suelen entregar una apreciación de lo realizado en la clase y luego analizan situaciones puntuales. Sin embargo, los contenidos de estas retroalimentaciones son difusos y tienden a ser emergentes, y sus prioridades son definidas principalmente por situaciones contingentes que viven los estudiantes. De esta forma, las interacciones se fundan en conversaciones genéricas e intuitivas. Los resultados muestran, también, que los contenidos de las retroalimentaciones tienden a estar más definidos cuando los acompañamientos se organizan de manera individual.

En quinto lugar, tanto el discurso como el análisis de las prácticas de acompañamiento relevan una importancia relativa, de carácter implícito y poco visible de las tributaciones entre las prácticas finales y los perfiles de egreso de cada carrera. Si bien en el análisis documental se aprecian competencias que tributan a la formación práctica, no es posible observar en las prácticas de acompañamiento ni en el discurso de los tutores y estudiantes la configuración de un objeto de análisis particular; más bien lo que se analiza es la acción, lo hecho, sin consideración de los supuestos involucrados y las consecuencias generadas. En el material analizado no es posible identificar el conjunto de categorías esenciales para emprender un análisis pedagógico, didáctico, profesional o socioprofesional. De otra parte, si bien emergen modalidades de acompañamiento diversas —individual, en tríadas y grupal—, lo más frecuente es el acompañamiento individual, y no se perciben diferencias sustantivas en la dinámica de la interacción entre los actores.

Entre los silencios del acompañamiento en la práctica final de la formación docente se encuentra la consideración, análisis y problematización de las experiencias y conocimientos previos de los futuros docentes acerca de la enseñanza escolar, y la distancia con procesos de investigación con perspectivas socioconstructivistas y foco en la experiencia práctica del futuro profesor. También están ausentes los hábitos metacognitivos, como los que describe Tardif (2012) a propósito de la reflexión sobre la experiencia práctica profesional. En suma, hay ausencia de modelamiento de los procesos de pensamiento ligados a la profesionalidad docente, lo cual resulta crítico. 
214 PRÁCTICAS DE ACOMPAÑAMIENTO DE PROFESORES TUTORES EN CARRERAS DE PEDAGOGÍA: ANÁLISIS DE LOS DISPOSITIVOS DE ASEGURAMIENTO Y CERTIFICACIÓN DE LA CALIDAD DE LOS APRENDIZAJES TERMINALES - M. Latorre, C. Vergara, M. Morales, A. Orbeta, C. Escobar, M. Quiroga

A casi 30 años del inicio de las reformas curriculares a los planes de estudio y currículos de formación inicial docente, es posible reconocer que la práctica y la formación práctica se ha instalado como un foco de atención y tensión en los programas de formación de futuros profesores en el país; sin embargo, los hallazgos de este estudio - aunque parciales y limitados- ponen en evidencia que aún queda mucho por hacer, y quizás entre lo más relevante esté la formación y especialización de quienes acompañan estos procesos, considerando nuevos marcos conceptuales y metodológicos para el desarrollo de dispositivos más pertinentes y acordes a la complejidad del objeto de análisis; además de la centralidad de la presencialidad como base de las prácticas de acompañamiento.

\section{Referencias}

Aguado, E. y Rogel, R. (2002). La recuperación del observador en la construcción del dato. Cinta moebio, 13, 2-20.

Ambrosetti, A. (2010). The Interconnectedness of the Roles of Mentorsand Mentees in Pre-service Teacher Education Mentoring Relationships. Australian Journal of Teacher Education, 35, 42-55.

Avalos, B. \& Aylwin, P. (2007). How young teachers experience their professional work in Chile. Teaching and Teacher Education, 23(4), 515-528.

Cochran-Smith, M. \& Lytle, S. (1999). Relationships of knowledge and practice: Teacher learning in communities. Review of Research in Education, 24, 249-305.

Correa, E. (2015). La alternancia en la formación inicial docente: vía de profesionalización. Educar, 51(2), 259-275.

Correa, E. (2014). Desafíos metodológicos para el estudio de la reflexión en contexto de formación docente. Estudios Pedagógicos, 40(1) (Número Especial), 71-86.

Correa, E. (2011). La práctica docente una oportunidad de desarrollo profesional. Perspectiva Educacional, 50 (2), 77-95.

Darling-Hammond, L. (2001). El derecho de aprender. Barcelona: Editorial Ariel Educación 
Darling-Hammond, L. (1994). Developing professional development schools: Early lessons, challenge, and promise Professional development schools: Schools for developing a profession. New York, NY: Teachers College Press.

De Ketele, JM. \& Roegiers, X. (1996). Méthodologie du recueil d’informations. Bruxelles-Paris: De Boeck Université.

Feiman Nemser, S. (2003). What New Teachers Need to learn. Educational Leadership, 60(8), 25-29.

Feiman Nemser, S. \& Buchmann, M. (1989). Pitfalls of experience in teacher preparation. Teacher College Record, 87(1), 53-65.

Hirmas, C. y Cortés, I. (2015). Estado del arte, Investigaciones sobre formación práctica en Chile. Tensiones y desafíos. Santiago de Chile: Organización de Estados Americanos para la Educación, la Ciencia y la Cultura.

Hmelo-Silver, C. E. (2004). Problem based learning: What and how learners learn? Educational Psychology Review, 16, 235-266.

Jyrhämä, R., Kynäslahti, H., Krokfors, L., Byman, R., Maaranen, K., Toom, A. $\&$ Kansanen, P. (2008). The appreciation and realisation of researchbased teacher education: Finnish students' experiences of teacher education. European Journal of Teacher Education, 31(1), 1-16.

Korthagen, F. (2004). In search of the essence of a good teacher: Towards a more holistic approach in teacher education. Teaching and Teacher Education, 20(1), 77-97.

Korthagen, F. \& Lagerwerf, B. (2001). Teachers' professional learning: how does it work? In: F. A. J. Korthagen, J. Kessels, B. Koster, B. Lagerwerf \& T. Wubbels, Linking practice and theory: The pedagogy of realistic teacher education (pp. 175-206). Mahwah: Lawrence Erlbaum Associates.

Lang, V. (1999). La professionnalisation des enseignants. Paris: Presses universitaires de France.

Lortie, D. (1975). School teacher: A sociological study. Chicago: University of Chicago Press.

Maes, M., Colognesi, A. \& Van Nieuwenhoven, A. (2018). «Accompagner/ former» ou «évaluer/vérifier». Education E Formation, 308, 95-106.

Maleyrot, E. (2016). La visée réflexive des dispositifs d'accompagnement à l'entrée dans le métier des enseignants du premier degré: étude diachronique par les missions des maîtres formateurs. Questions Vives, 24, 1-21 
216 PRÁCTICAS DE ACOMPAÑAMIENTO DE PROFESORES TUTORES EN CARRERAS DE PEDAGOGÍA: ANÁLISIS DE LOS DISPOSITIVOS DE ASEGURAMIENTO Y CERTIFICACIÓN DE LA CALIDAD DE LOS APRENDIZAJES TERMINALES - M. Latorre, C. Vergara, M. Morales, A. Orbeta, C. Escobar, M. Quiroga

Martín Sospedra, D. R. (2015). La formación docente universitaria en Cuba. Sus fundamentos desde una perspectiva desarrolladora del aprendizaje y la enseñanza. Estudios Pedagógicos, 41(1), 337-349.

Martinic, S., Moreno, R., Müller, M., Pimentel, F. Rittershaussen, S. Calderónf, M. y Cabezas, H. (2014). Análisis comparativo del componente de práctica en el currículo de formación profesional de médicos y profesores en la Pontificia Universidad Católica de Chile. Estudios Pedagógicos, 40(1), 179-196.

Martínez Diloné, H. y González Pons, S. (2010). Acompañamiento pedagógico y profesionalización docente: sentido y perspectiva. Revista Ciencia y Sociedad, 35(3), 521-541.

Mayoral-Serrat, P. y Castelló-Badia, M. (2015). Profesorado Novel y feedback del tutor. Un estudio de casos. Profesorado, 19(1), 346-362.

Mayring, P. (2003). Qualitative Inhaltsanalyse. Stuttgart: Weinheim und Basel.

Mercado Maldonado, R. (2010). Un debate actual sobre la formación inicial de docentes en México. Revista Semestral de Associaçao Brasileira de Psicologia Escolar e Educacional, 14(1) 149-157.

Montecinos, C., Barrios, C. y Tapia, MF. (2011). Relación entre estilos de supervisión durante la práctica profesional y las creencias de autoeficacia de los estudiantes de Pedagogía en Educación Básica. Revista Perspectiva Educacional, 50(2), 96-122.

Morales, Y. y Font, V. (2017). Análisis de la reflexión presente en las crónicas de estudiantes en formación inicial en Educación Matemática durante su periodo de práctica profesional. Acta Scientiae, 19(1), 122-137.

Murillo, J. (2006). Modelos innovadores en la formación inicial docente. Santiago de Chile: Unesco.

Ordóñez, G. y Ocampo, H. (2005). El acompañamiento tutorial como estrategia de la formación personal y profesional: Un estudio basado en la experiencia en una institución de educación superior. Universitas Psychologica, 4(1), 31-41.

Perez-Roux, T. (2016). Accompagnement des transitions professionnelles et dispositifs réflexifs en formation initiale et continue. Questions Vives, Recherches en éducation, 24.

Perrenoud, Ph. (2001). Développer la pratique réflexive dans le métier d'enseignant. Professionnalisation et raison pédagogique. Paris: ESF.

Perrenoud, Ph. (2005). Métier d'élève et sens du travail scolaire. Paris: ESF. 
Rebolledo, T. (2015). La formación inicial del profesorado de educación primaria y secundaria en Alemania, España, Finlandia, Francia y Reino Unido. Estudio comparado. Revista Española de Educación Comparada, 25, 129-148.

Rey, B. (2001). Manuels scolaires et dispositifs didactiques. En Y. Lenoir, B. Rey, G.-R. Roy \& J. Lebrun (Dir.), Le manuel scolaire et l'intervention éducative. Regards critiques sur ses apports et ses limites (pp. 25-40). Sherbrooke: Éditions du CRP de l'Université de Sherbrooke.

Richardson, V. \& Placier, P. (2001). Teacher change. In D.V. Richardson (Ed.), Handbook of research on teaching (4th ed) (pp. 905-950). Washington, DC: American Educational Research Association

Richardson, V. (1996). The role of attitudes and beliefs in learning to teach. In J. Sikula (Ed.), Handbook of research on teacher education (pp. 102119). New York: Simon \& Schuster Macmillan.

Rodríguez, D., Armengol, C. y Meneses, J. (2017). La adquisición de las competencias profesionales a través de las prácticas curriculares de la formación inicial de maestros. Revista de Educación, Ministerio de Educación, Cultura y Deporte, 376, 229-251.

Russell, T. (2012). Cambios paradigmáticos en la formación de profesores: peligros, trampas y la promesa no cumplida del profesional reflexivo. Encounters on Education, 13, 71-91.

Russell, T. (2014). La práctica en la formación de profesores: tensiones y posibilidades en la experiencia de aprender a enseñar. Estudios Pedagógicos, 49(1), 223-238.

Savoie-Zajc, L. (2004). La recherche qualitative/interprétative en éducation. En T. Karsenti y L. Savoie-Zajc (Eds.), La recherche en éducation: étapes et approches (pp. 122-151). Sherbrooke, Quebec: Éditions du CRP.

Schneuwly, B. (2002). L'écriture et son apprentissage: le point de vue de la didactique. Éléments de synthèse. Pratiques, 115/116, 237-246.

Solís, M. C., Núñez, C., Contreras, I., Rittershaussen, S., Montecinos, C. y Walker, H. (2011). Condiciones de la formación práctica de los futuros profesores. Estudios Pedagógicos, 38(1), 127-147.

Sutherland, L., Scanlon, L \& Sperring, A. (2005). New Directions in Preparing Professionals: Examining Issues in Engaging Students in Communities of Practice through a School-University Partnership. Teaching and Teacher Education: An International Journal of Research and Studies, 21(1), 79-92. 
218 PRÁCTICAS DE ACOMPAÑAMIENTO DE PROFESORES TUTORES EN CARRERAS DE PEDAGOGÍA: ANÁLISIS DE LOS DISPOSITIVOS DE ASEGURAMIENTO Y CERTIFICACIÓN DE LA CALIDAD DE LOS APRENDIZAJES TERMINALES - M. Latorre, C. Vergara, M. Morales, A. Orbeta, C. Escobar, M. Quiroga

Tardif, M. (2012). Réflexivité et expérience du travail enseignant : repenser le «praticien réflexif» à la lumière des traditions de la pensée réflexive. En M. Tardif, C. Borges \& A. Malo, Le virage réflexif en éducation (pp. 47-72). Bruselas: De Boeck.

Tardif, M. (2004). Los saberes del docente y su desarrollo profesional. Madrid: Narcea.

Tejada, J y Ruiz, C. (2013). Significación del prácticum en la adquisición de competencias profesionales que permiten la transferencia de conocimiento a ámbitos propios de la acción docente. Profesorado. Revista de currículum y formación del profesorado, 17(3), 91-110.

Yin, R. (2014). Case de study research: designs and methods. Thousand Oaks, CA: Sage.

Recibido: 26/12/2019

Aceptado: 25/09/2020 